\title{
Reconstructing Extraterrestrial Organic Synthesis via Site-Specific Isotope Measurements
}

\author{
TIMOTHY CSERNICA ${ }^{1}$, LAURA CHIMIAK ${ }^{1,2}$, SARAH \\ ZEICHNER $^{1}$, JASON DWORKIN ${ }^{3}$, JAMIE E ELSILA ${ }^{3}$ AND \\ JOHN EILER ${ }^{1}$ \\ ${ }^{1}$ Caltech \\ ${ }^{2}$ University of Colorado, Boulder \\ ${ }^{3}$ Goddard Space Flight Center \\ Presenting Author: tcsernic@caltech.edu
}

A key line of evidence in the search for life is the simple organic molecules - amino acids, sugars, and nucleobasesfound in all known life. There are many abiotic processes which create these molecules. Their presence in an asteroid, Martian sample, or other solar system body could result from life, productive abiotic processes that could lead to life, or unproductive processes that have nothing to do with life. To understand the organic material in a sample we must understand how it was formed.

Doing so is difficult because the same chemical structure can be formed via multiple routes. However, different synthesis pathways rely on different precursors and cause different isotope effects - the isotopic structure of a compound therefore encodes its synthetic history. By combining isotopic information from multiple compounds of interest, we can reconstruct the reactive pathways involved in a system.

Here, we present ongoing work to understand the organic synthesis that took place on Murchison meteorite via isotopic measurements of both meteorite organics and potential synthesis pathways. We are studying the site-specific isotopic structure of amino acids and adenine to track precursors to specific sites in these products. We are interpreting our measurements using the broad base of existing compound specific stable isotope data and laboratory chemical syntheses to generate a synthetic network describing prebiotic synthesis of biologically relevant organics in the chondritic meteorites.

Our results suggest that alanine and possibly other amino acids were formed through Strecker synthesis with precursor carbons coming from a ${ }^{13} \mathrm{C}$-rich $\mathrm{CO}$ pool and $\mathrm{CN}$ and $\mathrm{CH}_{\mathrm{x}}$ pools with ${ }^{13} \mathrm{C}$ content similar to bulk terrestrial and chondritic carbon. These same pools can be used to explain other meteorite organics, including amino acids, amines, and carboxylic acids. Our adenine measurements extend our network to nucleobases, relying on a mix of the $\mathrm{CO}$ and $\mathrm{CN}$ pools via a formamide/cyanide reaction.

Beyond informing our understanding of abiotic processes on meteorites, these experiments provide a test case for reconstruction of organic history on other solar system bodies, including Mars. We anticipate site-specific isotope analysis will be a useful tool for understanding organics throughout the solar system. 